\title{
Selectins and their counter receptors: a bitter sweet attraction
}

\author{
F A Symon, A J Wardlaw
}

A

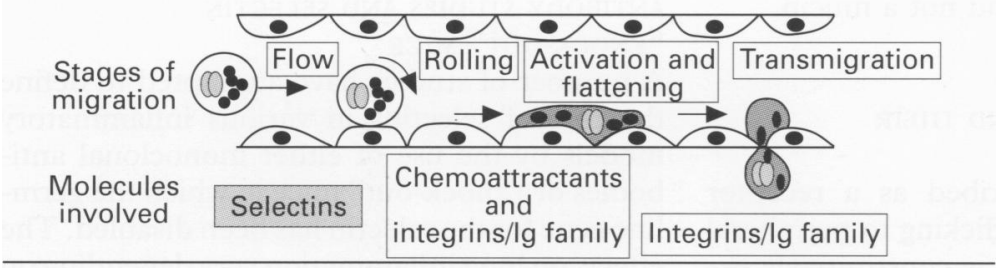

B

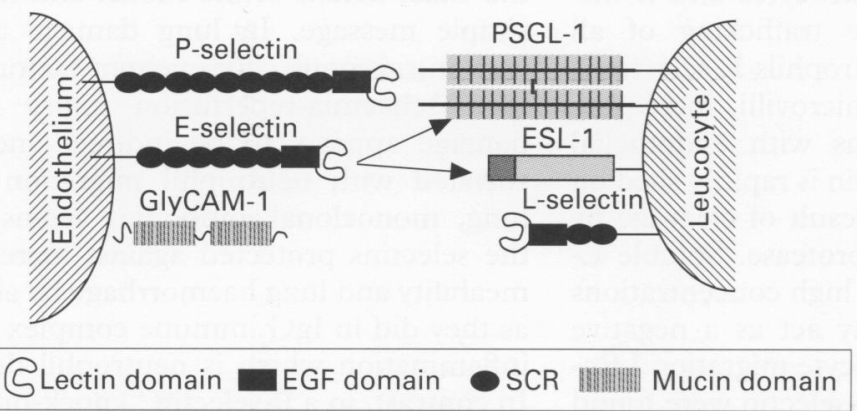

Figure 1 (A) Schematic outline of the stages involved in leucocyte migration through post-capillary venular endothelium. Leucocytes flowing in the vein become tethered to the vessel wall, a step mediated largely by the selectin family of adhesion receptors. The leucocytes then mll along the vessel wall until they come into contact with a

chemoattractant and become activated. Activation leads to engagement of members of the integrin family of receptors binding to immunoglobulin (Ig) family adhesion receptors on the endothelium. This causes them to become more firmly attached, stop rolling, flatten on the surface of the endothelial cell, and transmigrate. (B) Structure of the selectins and their ligands. E-selectin binds E-selectin ligand 1 (ESL-1) in mouse neutrophils, which is a chicken fibroblast receptor and not related to other adhesion receptor families. E-selectin also binds P-selectin glycoprotein 1 (PSGL-1) but with lower affinity than P-selectin. GlyCAM-1 lacks a transmembrane domain and may be secreted. Other L-selectin ligands not shown here are CD34 and mouse MAdCAM. EGF=epidermal growth factor;

$S C R=$ short consensus repeats.

Keywords: selectin, leucocytes, lung inflammation.

\begin{abstract}
Selectins are adhesion receptors expressed by leucocytes, platelets, and endothelial cells. They mediate the initial binding of leucocytes to vascular endothelium in the post-capillary venules. This is an essential first step in leucocyte migration into tissue. The selectin family of adhesion receptors consists of three $C$-type lectins ( $E$, $P$, and $L$ selectin). Their ligands (counter structures) are sialylated and fucosylated carbohydrate molecules which, in most cases, decorate mucin-like glycoprotein membrane receptors. Studies using blocking monoclonal antibodies have shown that inhibition of selectin function can ameliorate a range of inflammatory processes, offering the possibility that antagonists of selectin function may be useful in the treatment of inflammatory lung diseases such as asthma.

(Thorax 1996;51:1155-1157)
\end{abstract}

A number of lung diseases are thought to be due to inappropriate inflammatory responses. A central feature of inflammation is migration of leucocytes from the blood into tissue, a process controlled in part by adhesion molecules on the vascular endothelium and their counter receptors on leucocytes. Leucocyte migration is a sequential process. It is initiated by the tethering under flow conditions and subsequent rolling of leucocytes along the endothelial surface in post-capillary venules. This is followed by leucocyte activation leading to firm adhesion and flattening of the leucocytes against the vascular wall which, in turn, leads to extravasation. Each step in the sequence is necessary for migration to occur (fig 1A). ${ }^{1}$ The staged nature of migration allows considerable diversity of combination in the pattern of molecular signals required for cell trafficking. The selectins and their counter receptors are key receptors in the initial rolling interactions, capturing leucocytes under flow conditions and bringing them into close apposition with the vascular surface. While this model is assumed to apply to cell migration into the bronchial submucosa via the systemic bronchial circulation, migration into alveoli and bronchioles, which occurs largely through pulmonary capillaries, is less well defined and may involve a different set of signals. In particular, the small diameter of the capillaries and the pulsatile nature of flow in the pulmonary circulation suggest that arresting of leucocytes under flow, which is the primary role of the selectins, may not be a crucial step in migration into the alveoli.

\section{Scientific basis}

STRUCTURE OF SELECTINS AND THEIR COUNTER RECEPTORS

The selectins are a family of three closely related transmembrane glycoproteins - E, P, and L selectin. ${ }^{2}$ Their common structure consists of an $\mathrm{N}$-terminal $\mathrm{Ca}^{2+}$-dependent (C) lectin-type domain, an epidermal growth factor (EGF)like domain, multiple short consensus repeat (SCR) domains similar to those found in complement regulatory proteins, a transmembrane region, and a short cytoplasmic C-terminal domain. Together this arrangement results in an elongated structure which projects from the cell surface, ideal for initiating interactions with circulating leucocytes (fig 1B). The lectin domain forms the main ligand binding site, interacting with a carbohydrate determinant typified by fucosylated, sialylated, and usually sulphated glycans such as sialyl Lewis $\mathrm{X}$ 
(sLeX). The EGF domain may also play a part in ligand recognition. The multiple SCR domains (two for L-selectin, six for E-selectin, and nine for P-selectin) probably act as spacer elements, ensuring optimum positioning of the lectin and EGF domains for ligand interaction.

For high affinity binding the carbohydrate structures recognised by selectins need to be presented on a peptide backbone. This introduces a level of specificity. Most of the selectin glycoprotein counter receptors contain heavily O-glycosylated mucin-like domains. The potential mucin-like ligands for L-selectin have been defined as GlyCAM-1, CD34, and mouse (but probably not human) MAdCAM1. These receptors have been shown to play a part in lymphocyte recirculation (homing) but their importance in inflammation is uncertain. GlyCAM-1 lacks a transmembrane domain and it is uncertain whether it can act as a endothelial ligand for L-selectin. Functional studies have suggested that there is an inducible L-selectin counter receptor on endothelium but this remains to be defined, although in vitro human E-selectin has been shown to bind L-selectin.

P-selectin glycoprotein 1 (PSGL-1) is a counter receptor for P-selectin expressed by all leucocytes. ${ }^{3}$ It has a mucin-like domain although $\mathrm{N}$-terminal sulphated tyrosine residues are also important in binding. PSGL-1 binds E-selectin, but with lower affinity, and also possibly L-selectin where it may in part mediate neutrophil aggregation. The function of PSGL-1 appears to be controlled by differential glycosylation depending on the cell type or activation status of the cell on which it is expressed. An E-selectin ligand on mouse neutrophils has been identified as E-selectin ligand 1 (ESL-1). This was an isoform of a fibroblast growth factor and not a mucin.

\section{FUNCTION OF SELECTINS AND THEIR}

\section{EXPRESSION}

L-selectin was first described as a receptor mediating lymphocyte trafficking to peripheral lymph nodes. However, it is constitutively expressed by virtually all leucocytes and is important in mediating the trafficking of all leucocyte lineages. In neutrophils L-selectin is expressed on projecting microvilli, increasing the chance of interactions with endothelial counter receptors. L-selectin is rapidly shed on leucocyte activation as a result of cleavage by a membrane bound endoprotease. Soluble Lselectin can be detected in high concentrations in the blood where it may act as a negative feedback inhibitor of leucocyte migration. ${ }^{4} \mathrm{Re}-$ duced concentrations of $\mathrm{L}$-selectin were found in patients with the adult respiratory distress syndrome (ARDS) compared with normal controls and a group of at risk patients who did not progress to ARDS. The mechanism involved is obscure. $^{5}$

Unlike L-selectin, E-selectin and P-selectin are upregulated in response to inflammatory stimuli. P-selectin is constitutively stored in the Weibel-Palade bodies of endothelial cells and alpha granules in platelets. Within minutes of activation by inflammatory or thrombogenic mediators, such as thrombin or histamine, stored P-selectin is mobilised to the cell surface. In vitro studies in cultured human umbilical vein endothelial cells have shown this expression to be downregulated within minutes as a result of internalisation and degradation. ${ }^{6}$ Interleukin 3 can result in expression for up to four days. In vivo experiments studying leucocyte rolling in mouse cremaster muscle venules by intravital microscopy have indicated that Pselectin mediates the early rolling in response to the surgery (0-60 minutes), while at $60-120$ minutes rolling is dependent on L-selectin. Constitutive expression of P-selectin has been observed on vessels in rheumatoid synovium and on nasal polyp endothelium, suggesting a role in chronic inflammation. ${ }^{7}$

E-selectin is expressed by endothelial cells as a result of de novo protein synthesis in response to cytokine stimulation. It acts as a skin homing receptor for a subset of $T$ cells that express the cutaneous lymphocyte antigen (CLA) which contains an sLeX-like structure and is a ligand for E-selectin. CLA-positive T cells were greatly enriched in the skin of patients with atopic dermatitis but were largely absent from $T$ cells in the bronchoalveolar lavage fluid of asthmatic subjects. ${ }^{8}$ E-selectin can also mediate granulocyte binding to HUVEC. It is expressed in the airways of patients with asthma and rhinitis although no consistent difference from controls has been reported. Expression is induced after allergen challenge in both the airways and skin. ${ }^{9}$ Raised concentrations of soluble E-selectin have been detected in subjects with acute severe asthma but the levels did not correlate with disease activity.

ANTIBODY STUDIES AND SELECTIN

"KNOCK-OUT" MICE

A number of studies have attempted to define the role of selectins in various inflammatory models by the use of either monoclonal antibodies or "knock-out" mice in which the germline gene for the selectin has been disabled. The effects on lung inflammation vary depending on the exact details of the model and there is no simple message. In lung damage associated with intravenous cobra venom factor or a distant ischaemia-reperfusion injury in which damage appears to be indirect and not associated with neutrophil migration into the lung, monoclonal antibodies against each of the selectins protected against increased permeability and lung haemorrhage by about $50 \%$ as they did in IgG immune complex mediated inflammation which is neutrophil dependent. In contrast, in a P-selectin "knock-out" mouse no difference in neutrophil migration into the lung during Streptococcus pneumoniae induced pneumonia was observed compared with wild type mouse, whereas neutrophil migration in $S$ pneumoniae induced peritonitis was inhibited by about $70 \%$. An anti-E-selectin monoclonal antibody was also able to block neutrophil migration into the lung in a primate model of asthma and attenuate the late response. ${ }^{10} \mathrm{An}$ E-selectin "knock-out" mouse was healthy with little discernible abnormality in immune func- 
tion unless antibodies against P-selectin were also given. Consistent with this, a double mutant with deletion of both E- and P-selectin genes was severely immunocompromised although lung infection was not a significant feature. " The P- and L-selectin "knock-out" mice both had impaired neutrophil rolling and migration into the peritoneum after instillation of thioglycollate but, as mentioned above, no obvious impairment of lung immunity was found. In the L-selectin "knock-out" mice there was also atrophy of the peripheral lymph nodes consistent with its role as a peripheral lymphocyte homing receptor. The "knock-out" mice confirm the overlap in function between the selectins, particularly between E- and Pselectin. More models involving pulmonary inflammation need to be tested before a full understanding of the importance of selectins in lung immunity can be defined.

\section{Therapeutic potential}

Prevention of leucocyte migration into the lung may be useful in the treatment of a number of conditions ranging from asthma to fibrosing alveolitis. Selectins and their ligands are an attractive therapeutic target because their role, unlike many other adhesion receptors, appears largely confined to mediating leucocyte migration. The key role of the selectins in this function is illustrated by patients with the rare human type II leucocyte adhesion deficiency syndrome (LAD II) in which selectin dysfunction leads to impaired leucocyte migration and recurrent bacterial infections. ${ }^{12}$ As discussed above, studies in "knock-out" mice and those using anti-selectin monoclonal antibodies support the findings in LAD II by demonstrating impaired leucocyte rolling and $\mathrm{mi}-$ gration. The protection afforded by blocking selectin function in animal models of inappropriate inflammatory responses such as ischaemia-reperfusion support the concept of selectin antagonists as effective anti-inflammatory agents. However, from the relatively few models of lung inflammation that have been studied there is limited evidence that selectins are important in cell migration into the alveolar space so that, in the lung, airway diseases such as asthma may be the most appropriate target. There is clearly an overlap between the selectins in their key function of maintaining immunocompetence and this should protect against selectin antagonists causing significant immunosuppression. If individual selectins can be shown to have dominant roles in certain inflammatory lung diseases, then it should be possible to design safe and effective therapeutic agents. However, if two or more of the selectins need to be blocked to obtain efficacy, infectious complications may be a problem. The likely structure of selectin antagonists is uncertain. Sialylated oligosaccharides protected against lung injury in the P-selectin-dependent cobra venom model in rats, as well as myocardial reperfusion injury in cats. However, oligosaccharides have a low affinity for binding and are rapidly digested so they will need considerable modification before they can be used clinically. Antagonists directed against the peptide backbone of the selectin counter receptors may be a more fruitful approach.

\section{Conclusion}

Inappropriate inflammatory responses are an important cause of lung disease. The detailed dissection of the molecular basis of leucocyte migration now offers the hope that the inflammatory response can be selectively modulated without leading to unacceptable immunosuppression. The selectins and their counter structures are attractive targets because they control the first all-important step in leucocyte migration - the tethering of the leucocyte to the endothelium under flow. $\mathrm{Al}-$ though their structure is well characterised, our understanding of their individual role in controlling migration of leucocyte subsets in human disease is still patchy and it is unclear the extent to which their function overlaps. A challenge for the pharmaceutical industry is to design selectin antagonists which are effective but which avoid unacceptable immunosuppression. Topical therapy by inhalation may reduce this risk and asthma is an attractive therapeutic target. Mechanisms of migration into the alveolar compartment are relatively poorly understood and may not involve selectins. The extent to which selectin antagonists or other anti-adhesion strategies may be effective in treating interstitial lung disease is therefore uncertain.

1 Springer TA. Traffic signals for lymphocyte re-circulation and leukocyte emigration: the multi-step paradigm. Cell 1994;76:310.

2 Tedder TF, Steeber DA, Chen A, Engel P. The selectins: vascular adhesion molecules. FASEB f 1995;9:866-73.

3 Sako D, Chang XJ, Barone KM, et al. Expression cloning of a functional glycoprotein ligand for P-selectin. Cell 1993;75:1179-86.

4 Gearing AJ, Newman W. Circulating adhesion molecules in disease. Immunol Today 1993;14:506-12.

5 Donnelly SC, Haslett C, Dransfield I, Robertson CE, Carter DC, Ross JA, et al. Role of selectins in development of adult respiratory distress syndrome. Lancet 1994;344: 215-9.

6 Geng JG, Bevilacqua MP, Moore KL, et al. Rapid neutrophil adhesion to activated endothelium mediated by GMP140. Nature 1990;343:757-60.

7 Symon FA, Walsh GM, Watson S, Wardlaw AJ, Eosinophil adhesion to nasal polyp endothelium is P-selectin deadhesion to nasal polyp endothelium

8 Picker LJ, Martin RJ, Trumble AE, et al. Control of lymphocyte re-circualtion in man: differential expression of homing associated adhesion molecules by memory/effector $\mathrm{T}$ cells in pulmonary versus cutaneous effector sites. Eur $\mathcal{F}$ Immunol 1994;24:1269-77.

9 Montefort S, Gratziou C, Goulding D, Polosa R, Haskard DO, Howart PH, et al. Upregulation of leukocyte-endothelial cell adhesion molecules six hours after local allergen challenge of sensitised asthmatic airways. 7 Clin Invest 1993;93:1411-21.

10 Gundel RH, Weoner CD, Torcellini CA, Clarke CC, Haynes $\mathrm{N}$, Rothlein R, et al. ELAM-1 mediates antigen-induced $\mathrm{N}$, Rothlein $\mathrm{R}$, et al. ELAM-1 mediates antigen-induced acute airway inflammation and late phas

11 Frenette PS, Mayadas T, Rayburn H, Hynes RO, Wagner DD. Susceptibility to infection and altered hematopoiesis in mice deficient in both P- and E-selectins. Cell 1996; 84:563-74.

12 Etzioni A, Frydman M, Pollack S, Avidor I, Phillips ML, Paulson JC, et al. Severe recurrent chest infections due to a novel adhesion molecule defect. $N$ Engl f Med 1992; 327:1789-92. 